\title{
Detecção de Estilos de Aprendizagem utilizando Média Móvel Exponencialmente Ponderada
}

\author{
Patrick Ribeiro $^{1}$, Luciana Assis ${ }^{1}$, Alessandro Vivas ${ }^{1}$, Cristiano Pitangui ${ }^{2}$ \\ ${ }^{1}$ Programa de Pós-Graduação em Educação - PPGEd \\ Universidade Federal dos Vales do Jequitinhonha e Mucuri (UFVJM) \\ Diamantina - MG - Brasil \\ \{patrickalribeiro, lupassis, alessandro.vivas\}@gmail.com \\ ${ }^{2}$ Universidade Federal de São João del-Rei (UFSJ) \\ São João del-Rei - MG - Brasil. \\ pitangui.cristianodgmail.com
}

\begin{abstract}
This article proposes the using of Exponentially Weighted Moving Average (EWMA) in Learning Styles Detection process proposed by [Dorça 2012]. The aim of the use of EWMA in this process is to valorize the early results to make the algorithm detect the students' learning styles faster. The results and statistical analysis indicate that the method presented in this article can detect $L S$ faster than the other methods found in the literature.
\end{abstract}

Resumo. Este trabalho propõe a utilização do conceito de Média Móvel Exponencialmente Ponderada (MMEP) no processo de detecção e correção de Estilos de Aprendizagem (EAs) proposto por [Dorça 2012]. O objetivo da utilização desse conceito é valorizar bons resultados encontrados em sessões de aprendizagem anteriores de maneira a permitir que o algoritmo detecte os EAs do estudante mais rapidamente. Os resultados e análises estatísticas mostram que o método apresentado neste trabalho é capaz de detectar os EAs do estudante de forma mais rápida que os demais métodos encontrados na literatura.

\section{Introdução}

Os Estilos de Aprendizagem (EAs) de um indivíduo definem a forma como ele prefere receber e processar informação. Segundo [Felder and Silverman 1988] e [Lima and Catani 2015], eles são elementos determinantes para a qualidade do processo de aprendizagem.

Quando se trata de ensino presencial, é muito difícil estabelecer uma forma genérica de transmitir conhecimento e ao mesmo tempo abranger as especificidades de cada estudante. Assim, são raras as metodologias de ensino que abordam os EAs dos alunos [Felder and Silverman 1988, Felder and Henriques 1995, Coffield et al. 2004, Felder and Spurlin 2005].

Porém, a popularização do Ensino a Distância (EaD) e o avanço tecnológico têm colocado em evidência o conceito de EAs, que pode ser facilmente explorado em Ambientes Virtuais de Aprendizagem (AVAs). Tais ambientes são plataformas ou softwares desenvolvidos com o objetivo de monitorar e fazer gestão de conteúdos educacionais e 
VI Congresso Brasileiro de Informática na Educação (CBIE 2017)

Anais do XXVIII Simpósio Brasileiro de Informática na Educação (SBIE 2017)

do processo de aprendizagem do estudante, além de permitir a comunicação entre os usuários, o acompanhamento do progresso do estudante dentro do curso, dentre outros [Cavus and Alhih 2014, Cavus 2015].

Com a introdução dos EAs, os AVAs passam a ter a capacidade de fornecer o conteúdo de forma personalizada. Surgem, então, os Sistemas de Tutoria Inteligentes (STI), cujo objetivo, segundo [Brusilovsky and Millán 2007], é identificar as preferências do estudante e, com base nelas, fornecer o conteúdo de forma adaptada.

Nesse sentido, diferentes abordagens apresentam métodos para identificação automática de EAs. [Sena et al. 2016], por exemplo, utilizam o conceito de Modelos Ocultos de Markov e o Modelo de EAs de Felder e Silverman (FSLSM). Por outro lado, [Dorça 2012] propôs e validou, por meio de simulação, um algoritmo capaz de identificar e corrigir os EAs em Sistemas Inteligentes Adaptativos para Educação, utilizando Aprendizado por Reforço (AR), Cadeias de Markov (CM), Taxonomia de Bloom e o FSLSM.

Com o intuito de detectar EAs de forma mais eficiente, este artigo propõe o desenvolvimento de um método baseado em Média Móvel Exponencialmente Ponderada (MMEP) combinada com AR em um ambiente que simula o comportamento de um aluno em um AVA, proposto por [Dorça 2012].

Adiante, a seção 2 apresenta a fundamentação teórica para o desenvolvimento do trabalho. A seção 3 descreve o funcionamento do algoritmo desenvolvido. Na seção 4 são apresentados os resultados computacionais. Por fim, a seção 5 expõe as considerações finais e perspectivas de trabalhos futuros.

\section{Fundamentação teórica}

\subsection{Estilos de Aprendizagem}

[Felder and Silverman 1988] definem Estilos de Aprendizagem (EAs) como a forma através da qual o indivíduo melhor recebe e processa a informação. Nesse sentido, saber quais são os estilos do estudante pode melhorar seu processo de aprendizagem, pois, com base nessas informações, a melhor estratégia pedagógica pode ser adotada.

Sabendo que essas preferências são individuais, diversos estudiosos apresentaram modelos que pudessem identificá-las. Esses modelos são conhecidos como Modelos de Estilos de Aprendizagem e, em seu estudo, [Coffield et al. 2004] contabilizam mais de 70 deles.

O Modelo de Estilos de Aprendizagem de Felder e Silverman (FSLSM) é um dos mais utilizados na literatura. Um dos motivos para essa ampla utilização se dá pela existência do Índice de Estilos de Aprendizagem (ILS), ferramenta utilizada para identificar os EAs do aluno [Silva 2012]. O modelo aplicado neste trabalho é o FSLSM, detalhado na seção 2.2 .

\subsection{Modelo de Estilos de Aprendizagem de Felder e Silverman}

O FSLSM foi proposto, inicialmente, com 5 dimensões, onde cada dimensão possuía 2 EA. Depois de alguns ajustes, [Felder and Henriques 1995] apresentaram o modelo da forma como se encontra atualmente: 8 EA distribuídos 2 a 2 em 4 dimensões. A organização dessas dimensões e seus respectivos EA pode ser visualizada na Figura 1. 
VI Congresso Brasileiro de Informática na Educação (CBIE 2017)

Anais do XXVIII Simpósio Brasileiro de Informática na Educação (SBIE 2017)

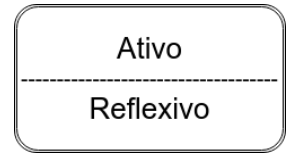

(a) Processamento

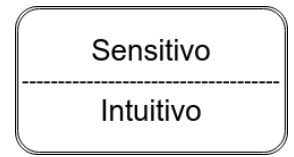

(b) Percepção

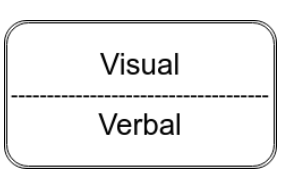

(c) Entrada

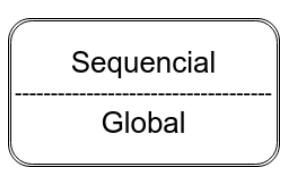

(d) Organização

Figure 1. Estrutura do FSLSM

Uma característica do FSLSM que o difere de muitos outros modelos é que o estudante pode possuir preferências por 2 EA em uma mesma dimensão. Porém, essa preferência é sempre mais intensa por um EA do que a outro, sendo essas preferências complementares entre si.

Considerando a dimensão Processamento, o estudante pode preferir aprender ou em grupo (EA ativo) ou individualmente (EA reflexivo). Quanto à dimensão Percepção, o estudante pode preferir aprender através de fatos concretos, tais como exemplos, (EA sensitivo) ou por meio de abstrações como teorias e equações (EA intuitivo). A dimensão Entrada apresenta o EA visual, que se refere a estudantes que preferem aprender por meio da visão (gráficos, imagens, etc.), e o EA verbal que é atribuído aos estudantes que têm como preferência o aprendizado por meio da linguagem falada ou escrita. Por fim, os estudantes com EA sequencial preferem aprender de forma progressiva através de etapas bem definidas. Ao contrário, os estudantes com o EA global preferem aprender de forma livre, sem a necessidade de uma sequência lógica [Felder and Silverman 1988, Felder and Henriques 1995, Dorça et al. 2011].

Conforme mencionado, o ILS permite a identificação desses estilos. Essa ferramenta é um questionário com 44 questões, sendo 11 para cada dimensão do FSLSM. Os estilos são identificados a partir da quantidade de questões respondidas a seu favor [Silva 2012].

A diferença entre as quantidades dos estilos de uma dimensão define a força do EA com mais respostas a seu favor. Caso essa diferença seja 1 ou 3, o EA é classificado como leve. Caso seja igual a 5 ou 7, ele é classificado como moderado. Caso seja 9 ou 11, o EA é classificado como forte.

A validade do ILS foi objeto de estudo de [Felder and Spurlin 2005], que chegaram à conclusão de que a ferramenta é capaz de identificar com certo grau de confiabilidade e precisão os EA de um estudante. Contudo, a aplicação desse tipo de ferramenta de forma manual torna o processo de detecção vulnerável, já que a identificação é feita apenas no início e as preferências do estudante podem mudar ao longo do processo. Assim, a melhor forma de se detectar EA de forma precisa é através de métodos automáticos e dinâmicos.

\subsection{Abordagem estocástica e dinâmica para detecção e correção de EA}

[Dorça 2012] desenvolveu um modelo cujo objetivo é detectar os EA de um estudante. A ferramenta proposta pelo autor é composta por 3 módulos: Modelo do Estudante (ME), Módulo Pedagógico (MP) e Componente de Modelagem do Estudante (CME).

$\mathrm{O}$ ME armazena informações sobre o estudante, como os EA inferidos pelo sistema, o conceito que está sendo estudado na sessão de aprendizagem e o nível cognitivo 
VI Congresso Brasileiro de Informática na Educação (CBIE 2017)

Anais do XXVIII Simpósio Brasileiro de Informática na Educação (SBIE 2017)

do estudante. O EA do estudante é atualizado quando a performance obtida pelo estudante é inferior ao mínimo exigido pelo sistema, 60 , de modo que ao término do processo os EA inferidos pelo sistema refletem os EA reais do estudante.

No FSLSM cada dimensão possui $2 \mathrm{EA}$, de modo que a pessoa pode ter preferência por ambos os estilos, mas sempre um prevalecendo sobre o outro. Essas preferências são representadas por probabilidades e o nível cognitivo por valores de 1 a 6 . Esses valores representam os 6 níveis hierárquicos da taxonomia proposta por [Bloom et al. 1956] e revisada por [Krathwohl 2002].

As probabilidades dos EAs de uma mesma dimensão são complementares. Dessa forma, ao incrementar a probabilidade de um EA, deve-se decrementar a probabilidade do outro EA.

O MP contém as estratégias pedagógicas a serem adotadas para apresentar um determinado conteúdo. Nesse caso, cada estratégia é representada pela combinação de 4 EAs, sendo cada EA de uma dimensão do FSLSM. Assim, tem-se $16\left(2^{4}\right)$ Combinações de Estilos de Aprendizagem (CEAs).

Dadas as 16 CEAs, o MP seleciona uma através de Cadeias de Markov (CM). Para cada dimensão é definida uma $\mathrm{CM}$ e sua mudança de estado é definida pelas probabilidades de cada EA armazenadas no ME. A $C E A=$ $\{$ Ativo, Sensitivo, Visual, Sequencial $\}$, por exemplo, tem sua probabilidade de ocorrência dada por:

$$
\operatorname{Prob}_{C E A}=\operatorname{Prob}_{\text {Ativo }} \times \operatorname{Prob}_{\text {Sensitivo }} \times \operatorname{Prob}_{\text {Visual }} \times \operatorname{Prob}_{\text {Sequencial }}
$$

O Componente de Modelagem do Estudante é responsável por promover ajustes no Modelo do Estudante. A cada sessão de aprendizagem, o desempenho do estudante é analisado e, caso seja inferior ao mínimo exigido pelo sistema (60), um sinal de reforço, multipicado pela taxa de aprendizagem do agente (0.5), é aplicado sobre as probabilidades dos EA presentes no ME. Esse reforço é baseado no algoritmo de Aprendizagem por Reforço Q-Learning e seu cálculo é feito a partir da equação 2.

$$
R=\frac{1}{P F M \times D E A}
$$

Em que:

- $R$ é o valor do reforço que se deseja obter;

- $P F M$ é a nota recebida pelo estudante ao final da sessão de aprendizagem;

- $D E A$ é a diferença entre as probabilidades de ocorrência dos EA de uma dimensão que compõem o ME.

A verificação e validação da ferramenta proposta foi feita através de um simulador chamado Processo Estocástico de Simulação de Desempenho do Estudante (PESDE), também desenvolvido por [Dorça 2012]. Ao final de cada sessão de aprendizagem, uma nota é gerada a partir da equação 3 . Caso essa nota seja inferior a 60, o reforço é aplicado às probabilidades dos EA no ME. Além disso, o estudante permanece no mesmo nível 
VI Congresso Brasileiro de Informática na Educação (CBIE 2017)

Anais do XXVIII Simpósio Brasileiro de Informática na Educação (SBIE 2017)

cognitivo (considerando a Taxonomia de Bloom) e o conceito é apresentado novamente. Caso o estudante obtenha uma nota satisfatória, o seu nível cognitivo é incrementado. Ao atingir o nível cognitivo máximo, outro conceito é apresentado ao aluno.

$$
P F M=100-(Q P N S \times K \times \beta)
$$

Na qual:

- $Q P N S$ representa a quantidade de preferências não satisfeitas, ou a quantidade de preferências que não coincidem entre a CEA selecionada e a CEA real do estudante;

- $K$ é uma constante de valor 20 definida pelo autor;

- $\beta$ é o fator aleatório que representa a estocasticidade do processo e cujo valor está o intervalo $[0,1]$.

\section{Abordagem proposta}

Apesar de bem desenvolvida, a proposta de [Dorça 2012] tem, na frequência dos ajustes dos EA probabilísticos, uma fragilidade. $\mathrm{O}$ fato de ajustar com grande frequência o $\mathrm{ME}$ pode implicar em uma demora na detecção dos EA. Isso porque a ferramenta proposta é fortemente influenciada por fatores externos.

Outras propostas já foram apresentadas no sentido de tornar o método desenvolvido por [Dorça 2012] mais eficiente, como é o caso de [Rodrigues et al. 2016], que buscam aprimorar a seleção das estratégias pedagógicas substituindo o mecanismo de Cadeias de Markov por Lógica Fuzzy. Além de [Rodrigues et al. 2016], [Gonçalves et al. 2016] e [Falci et al. 2017] também apresentaram propostas nesse sentido.

Com o intuito de reduzir o tempo gasto para se detectar EA, este artigo apresenta um método que utiliza o conceito de Média Móvel Exponencialmente Ponderada (MMEP) para decidir quando ajustar o ME. Com isso os ajustes serão mais precisos reduzindo a quantidade de sessões de aprendizagem necessárias para que ocorra detecção dos EAs do estudante.

\subsection{Média Móvel Exponencialmente Ponderada}

A Média Móvel Exponencialmente Ponderada (MMEP) utiliza um fator de suavização para o cálculo de uma média diferenciada, sendo comumente utilizada nas áreas de finanças, monitoramento e ajuste de processos e análises de séries temporais. Sua fórmula geral é dada pela equação 4 [Box et al. 2005, Awheda and Schwartz 2013].

$$
\hat{y}_{t+1}=\lambda y_{t}+\theta \hat{y}_{t}
$$

Na qual:

- $\theta$ é o fator de suavização;

- $\lambda$ é $1-\theta$;

- $\hat{y}_{t+1}$ é a previsão que se deseja fazer (MMEP das notas);

- $y_{t}$ é o resultado encontrado atualmente (nota); 
VI Congresso Brasileiro de Informática na Educação (CBIE 2017)

Anais do XXVIII Simpósio Brasileiro de Informática na Educação (SBIE 2017)

- $\hat{y}_{t}$ é a previsão feita anteriormente (MMEP calculada na sessão de aprendizagem anterior).

Esse recurso tem a capacidade de suavizar curvas com base em resultados ocorridos anteriormente. Essa capacidade é vista como uma oportunidade de impedir que resultados ruins, oriundos de fatores externos, destruam uma tendência construída há algum tempo.

As modificações propostas neste trabalho foram aplicadas ao Modelo do Aluno, Módulo Pedagógico e ao Componente de Modelagem do Estudante.

\subsection{Modelo do Estudante}

A estrutura do ME é apresentada na tabela 1. Este modelo é similar ao apresentado por [Dorça 2012], porém, para utilização das MMEP, o Modelo do Estudante passa a armazenar, também, as MMEP das notas do aluno. Assim, a cada sessão de aprendizagem, a MMEP das notas do estudante, para cada CEA, é armazenada no Módulo do Estudante (ME).

Tabela 1. Modelo do Estudante proposto por [Dorça 2012] e utilizado neste artigo

\begin{tabular}{|c|c|c|c|c|c|c|c|}
\hline \multicolumn{8}{|c|}{ EA Probabilísticos } \\
\hline \multicolumn{2}{|c|}{ Processamento } & \multicolumn{2}{|c|}{ Percepção } & \multicolumn{2}{|c|}{ Entrada } & \multicolumn{2}{|c|}{ Organização } \\
\hline Ativo & Reflexivo & Sensitivo & Intuitivo & Visual & Verbal & Sequencial & Global \\
\hline 0,35 & 0,65 & 0,17 & 0,83 & 0,89 & 0,11 & 0,84 & 0,16 \\
\hline \multicolumn{8}{|c|}{ Objetivos de Aprendizagem e Estado Cognitivo } \\
\hline \multicolumn{8}{|c|}{$\begin{array}{c}O A=\left\{\left\langle C_{0}, 5\right\rangle \ldots\left\langle C_{n-1}, 5\right\rangle\right\} \\
E C=\left\{\left\langle C_{0},-1\right\rangle \ldots\left\langle C_{n-1},-1\right\rangle\right\}\end{array}$} \\
\hline & & \multicolumn{4}{|c|}{ MMEP } & & \\
\hline & & \multicolumn{3}{|l|}{ CEA } & MMEP & & \\
\hline & & \multicolumn{3}{|c|}{$C E A_{1}=\{A t i$, Sen, Vis, Seq $\}$} & 89 & & \\
\hline & & \multirow{2}{*}{\multicolumn{3}{|c|}{$\begin{array}{l}C E A_{2}=\{A t i, \text { Sen }, \text { Vis }, \text { Glo }\} \\
C E A_{3}=\{A t i, \text { Sen }, \text { Ver }, \text { Seq }\}\end{array}$}} & 81 & & \\
\hline & & & & & & & \\
\hline & & \multicolumn{3}{|c|}{$\ldots$} & & & \\
\hline & & \multicolumn{3}{|c|}{$C E A_{14}=\{\operatorname{Ref}$, Int, Vis, Glo $\}$} & 71 & & \\
\hline & & \multirow{2}{*}{\multicolumn{3}{|c|}{$C E A_{15}=\{R e f$, Int, Ver, Seq $\}$}} & 0 & & \\
\hline & & $C E A_{16}=\{\operatorname{Ref}$, Int, Ver, Glo $\}$ & & & 87 & & \\
\hline
\end{tabular}

O cálculo da MMEP das notas é baseado na equação 4. Nela, $y_{t}$ é substituído pela nota obtida pelo estudante na sessão de aprendizagem atual e $\hat{y}_{t}$ consiste no valor da MMEP, armazenado no ME.

\subsection{Módulo Pedagógico}

O Modelo Pedagógico deste trabalho segue as ideias apresentadas por [Dorça 2012]. Para cada conteúdo a ser apresentado e, para cada nível cognitivo, o sistema seleciona uma CEA, que irá representar a estratégia pedagógica a ser adotada naquela sessão de aprendizagem. Para selecionar uma CEA, o sistema utiliza Cadeias de Markov para cada dimensão do FSLSM, cuja mudanças de estado é dada pelos EAs probabilísticos armazenados no ME. Em seguida, o sistema avalia o desempenho do aluno, com base na estratégia de aprendizagem adotada. Esse procedimento é executado pelo PESDE seguindo a equação 3 apresentada na seção 2.3 . 
VI Congresso Brasileiro de Informática na Educação (CBIE 2017)

Anais do XXVIII Simpósio Brasileiro de Informática na Educação (SBIE 2017)

\subsection{Componente de Modelagem do Estudante}

O Componente de Modelagem do Estudante, proposto por [Dorça 2012], verifica se a nota (desempenho) obtida pelo estudante foi inferior 60, se sim, ajustes são feitos no ME. Por outro lado, na proposta de [Gonçalves et al. 2016], além dos desempenhos ruins, os desempenhos de excelência, configurados conforme indicação pedagógica, são parâmetros para ajustes no ME.

Neste trabalho, a verificação é feita com base na nota do estudante e com base na MMEP das notas, de modo que o ME apenas é ajustado quando ambas forem inferior a 60. Assim, ao final de cada sessão de aprendizagem a MMEP é calculada a partir da equação 4. Nela, $y_{t}$ é substituído pela nota e $\hat{y}_{t}$ é substituído pelo valor da MMEP da última sessão de aprendizagem em que a atual CEA foi selecionada.

Caso ambas sejam inferior a 60 as probabilidades dos EAs Probabilísticos são modificadas conforme as regras propostas por [Dorça 2012]. Caso apenas a nota seja inferior a 60, não se aplica mudanças no ME, porém o estudante repete a sessão de aprendizagem. Caso a nota do estudante seja igual ou superior a 60, nada é feito e o estudante progride no curso.

Os ajustes são realizados de modo a decrementar as probabilidades dos EAs que compõem a CEA selecionada, e incrementar as probabilidades dos seus complementares. Para isso é utilizado o valor do reforço conforme propõe [Dorça 2012] na equação 2, e a taxa de aprendizagem do agente que, diferente do que propõe o autor, neste trabalho varia em função da nota do estudante. A equação 5 define o valor da taxa de aprendizagem do agente ao final de cada sessão de aprendizagem.

$$
\alpha=1-\left(\frac{P F M}{100}\right)
$$

\section{Resultados computacionais}

Os testes foram feitos a partir da PESDE, plataforma de simulação desenvolvida por [Dorça 2012]. A primeira etapa da fase de testes consistiu em definir qual o melhor valor para o fator de suavização $(\theta)$ (ver equação 4). Para a realização dos testes, considerou-se o ME definido sempre da mesma forma, variando, portanto, o perfil de estudante que utilizou o sistema (EA real). Assim, depois de executar 30 vezes cada EA real, com o valor de $\theta$ variando de 0.1 a 0.9 , chegou-se à conclusão de que os melhores resultados foram obtidos $\operatorname{com} \theta=0.1$.

A partir de então, os resultados obtidos para cada perfil de estudante foram comparados com os resultados obtidos pelo algoritmo proposto por [Dorça 2012]. Lembrando que ambos algoritmos foram testados sob as mesmas condições iniciais. A Tabela 2 mostra as quantidades médias de sessões de aprendizagem necessárias para que cada abordagem detectasse os EAs do estudante, ao final das 30 simulações. Foram 50 conceitos apresentados, considerando os 6 níveis cognitivos, totalizando 300 sessões de aprendizagem. Porém, ao obter uma nota insatisfatória, o sistema repete a sessão de aprendizagem até obter nota satisfatória. Assim sendo, uma menor quantidade de sessões de aprendizagem é um indício de uma apresentação mais adequada do conteúdo, dada a detecção mais eficiente do EA. 
VI Congresso Brasileiro de Informática na Educação (CBIE 2017)

Anais do XXVIII Simpósio Brasileiro de Informática na Educação (SBIE 2017)

A Tabela 2 mostra em negrito o método que obteve, para cada perfil de estudante simulado, a menor quantidade média de sessões de aprendizagem. Isso representa aquele que levou o menor tempo médio para identificar os EAs.

Pode-se observar que, dos 16 perfis simulados, em 10 a quantidade média de sessões de aprendizagem obtida pelo algoritmo aqui proposto foi menor do que a quantidade média de sessões de aprendizagem gerada pelo método encontrado na literatura.

Isso significa que, ao final de 30 simulações de 10 dentre 16 perfis de estudante, o tempo médio gasto para identificar as preferências do estudante foi menor na abordagem aqui apresentada quando comparada com a abordagem de 2012.

Tabela 2. Quantidades médias de sessões de aprendizagem por perfil simulado

\begin{tabular}{|c|c|c|}
\hline CEA & Dorça & Ribeiro \\
\hline$C E A_{1}=\{A t i$, & 326,36 & 0 \\
\hline$C E A_{2}=\{A t i$, Sen, Vis, Glo $\}$ & & 325,46 \\
\hline$C E A_{3}=\{A t i$, Sen, Ver, Seq $\}$ & 6 & 329,2 \\
\hline$C E A_{4}=\{A t i$, Sen, Ver, Glo $\}$ & 33,1 & 429,3 \\
\hline$C E A_{5}=\{A t i$, Int, Vis, Seq $\}$ & & 38,6 \\
\hline$C E A_{6}=\{A t i$, Int, Vis, Glo $\}$ & 1 & 426,73 \\
\hline$C E A_{7}=\{A t i$, Int, Ver, Seq $\}$ & & 86 \\
\hline$C E A_{8}=\{$ Ati, Int, Ver, Glo $\}$ & & 16,9 \\
\hline$C E A_{9}=\{\operatorname{Ref}$, Sen, Vis, Seq $\}$ & 360,93 & 329,06 \\
\hline$C E A_{10}=\{\operatorname{Ref}$, Sen, Vis, Glo $\}$ & 2 & 431,1 \\
\hline$C E A_{11}=\{\operatorname{Ref}$, Sen, Ver, Seq $\}$ & 4 & 428,96 \\
\hline$C E A_{12}=\{\operatorname{Ref}$, Sen, Ver, Glo $\}$ & & 5,26 \\
\hline$C E A_{13}=\{\operatorname{Ref}$, Int, Vis, Seq $\}$ & 28,8 & 427,9 \\
\hline$C E A_{14}=\{\operatorname{Ref}$, Int, Vis, Glo $\}$ & 316 & 317,4 \\
\hline$C E A_{15}=\{\operatorname{Ref}$, Int, & 314,23 & 317,43 \\
\hline$C E A_{16}=\{\operatorname{Ref}$, Int, Ver, Glo $\}$ & 308,33 & 308,6 \\
\hline
\end{tabular}

A partir dos dados gerados por ambas as abordagens, obteve-se 2 grupos com 480 valores cada (16 perfis simulados 30 vezes), sendo cada grupo proveniente de uma abordagem. Sobre ambos os grupos foi aplicado o teste de normalidade de Shapiro-Wilk, que permitiu concluir que os dados não seguem a distribuição normal.

Assim, optou-se por submeter as amostras ao teste não paramétrico MannWhitney. Esse teste foi escolhido devido à natureza dos conjuntos de dados coletados. Como os conjuntos são oriundos de dois algoritmos distintos e esses conjuntos não afetam um ao outro, os dados são caracterizados independentes [Larson and Farber 2010, p.352].

Como resultado do teste de Mann-Whitney, pode-se rejeitar, com base no p-valor obtido (0.04861), ao nível de confiança de 0.95, a hipótese de que a quantidade de sessões de aprendizagem do algoritmo proposto por [Dorça 2012] é menor ou igual à quantidade de sessões de apredizagem do algoritmo proposto neste trabalho. Esse resultado, apesar de muito próximo do nível de significância de 0.05 , reforça as considerações feitas com base nos dados da Tabela 2, em que, para a maioria dos perfis simulados, esta abordagem 
VI Congresso Brasileiro de Informática na Educação (CBIE 2017)

Anais do XXVIII Simpósio Brasileiro de Informática na Educação (SBIE 2017)

proporcionou uma média de sessões de aprendizagem menor do que a abordagem presente na literatura.

\section{Considerações finais}

Fornecer o conteúdo ao estudante de forma adaptada às suas preferências não é uma tarefa fácil devido ao grau de incerteza envolvido no processo. Mesmo não sendo simples, [Dorça 2012] propôs um sistema que simule esse processo levando em consideração diversos fatore que podem influenciar no desempenho do aluno e simular o processo de detecção do EA do aluno. Entretanto, os fatores que não estão associados às preferências do estudante e as estratégias pedagógicas utilizadas para apresentar o conteúdo têm grande influência nos ajustes do sistema, o que pode prejudicar o processo de detecção. A abordagem aqui proposta mostrou-se capaz de realizar a detecção dos EA do estudante com maior rapidez quando comparada com o método proposto por [Dorça 2012], uma vez que ele é menos sensível as intempéres inerentes ao sistema. Trabalhos futuros apontam para uma aplicação real do método proposto em um Ambiente Virtual de Aprendizagem para validar o método aqui apresentado.

\section{Referências}

Awheda, M. D. and Schwartz, H. M. (2013). Exponential Moving Average Q-Learning Algorithm. IEEE Symposium on Adaptive Dynamic Programming and Reinforcement Learning, ADPRL, pages 31-38.

Bloom, B. S., Engelhart, M. D., Furst, E. J., Hill, W. H., and Krathwohl, D. R. (1956). Taxonomy of Educational Objectives: The Classification of Educational Goals. David McKay Company Inc.

Box, G. E. P., Hunter, J. S., and Hunter, W. G. (2005). Statistics for Experimenters: Design, Innovation and Discovery. John Wiley \& Sons, Inc., Hoboken, New Jersey, 2nd ed. edition.

Brusilovsky, P. and Millán, E. (2007). User Models for Adaptive Hypermedia and Adaptive Educational Systems. The Adaptive Web, pages 3-53.

Cavus, N. (2015). Distance Learning and Learning Management Systems. Procedia Social and Behavioral Sciences, 191:872-877.

Cavus, N. and Alhih, M. S. (2014). Learning management systems use in science education. Procedia - Social and Behavioral Sciences, 143:517-520.

Coffield, F., Moseley, D., Hall, E., and Ecclestone, K. (2004). Learning styles and pedagogy in post-16 learning A systematic and critical review. Technical report.

Dorça, F. A. (2012). Uma Abordagem Estocástica Baseada em Aprendizagem por Reforço para Modelagem Automática e Dinâmica de Estilos de Aprendizagem de Estudantes em Sistemas Adaptativos e Inteligentes para Educação a Distância. Tese (Doutorado), Universidade Federal de Uberlândia.

Dorça, F. A., Lima, L. V., Fernandes, M. A., and Lopes, C. R. (2011). Detecção e Correção Automática de Estilos de Aprendizagem em Sistemas Adapativos para Educação. Revista de Informática Teórica e Aplicada, 18:178-204. 
VI Congresso Brasileiro de Informática na Educação (CBIE 2017)

Anais do XXVIII Simpósio Brasileiro de Informática na Educação (SBIE 2017)

Falci, S. H., Vivas, A., Assis, L., and Pitangui, C. (2017). Uma Nova Abordagem para Aplicação de Reforço em Sistemas Automáticos e Adaptativos de Detecção de Estilos de Aprendizagem. Revista Eletrônica Argentina-Brasil de Tecnologias da Informação e da Comunicação, 1(6).

Felder, R. and Silverman, L. (1988). Learning and teaching styles in engineering education. Engineering education, 78(June):674-681.

Felder, R. M. and Henriques, E. R. (1995). Learning and Teaching Styles In Foreign and Second Language Education. Foreign Language Annals, 28(1):21-31.

Felder, R. M. and Spurlin, J. (2005). Applications, Reliability and Validity of the Index of Learning Styles*. International Journal of Engineering Education, 21(1):103-112.

Gonçalves, A. V., Vivas, A., Assis, L., Pitangui, C., and Dorça, F. (2016). Avanços na modelagem automática e dinâmica de estilos de aprendizagem de estudantes em sistemas adaptativos e inteligentes para educação: uma análise experimental. In XXVII Simpósio Brasileiro de Informática na Educação (SBIE 2016), pages 1006-1015, Uberlândia. Sociedade Brasileira de Computação - SBC.

Krathwohl, D. R. (2002). A Revision of Bloom's Taxonomy: An Overview. Theory Into Practice, 41(4):212-218.

Larson, R. and Farber, B. (2010). Estatística aplicada. Pearson Education do Brasil, São Paulo, 4 edition.

Lima, A. L. G. and Catani, D. B. (2015). "Que tipo de aluno é esse ?”: psicologia , pedagogia e formação. Revista Brasileira de Educação, 20(62):571-594.

Rodrigues, L. H. S., Assis, L., Vivas, A., Pitangui, C., and Falci, S. (2016). Uso de Lógica Fuzzy na Seleção de Estratégias de Aprendizagem. In XXVII Simpósio Brasileiro de Informática na Educação (SBIE 2016), pages 1076-1085, Uberlândia. Sociedade Brasileira de Computação - SBC.

Sena, E., Vivas, A., Assis, L., and Pitangui, C. (2016). Proposta de uma Abordagem Computacional para Detecção Automática de Estilos de Aprendizagem Utilizando Modelos Ocultos de Markov e FSLSM. In XXVII Simpósio Brasileiro de Informática na Educação (SBIE 2016), page 1126, Uberlândia. Sociedade Brasileira de Computação SBC.

Silva, L. L. V. (2012). Estilos e estratégias de aprendizagem de estudantes universitários. Dissertação (Mestrado), Instituto de Psicologia da Universidade de São Paulo, São Paulo. 